\title{
Antidepressant Agent
}

National Cancer Institute

\section{Source}

National Cancer Institute. Antidepressant Agent. NCI Thesaurus. Code C265.

A pharmacological agent that acts on neurotransmitter signaling pathways to alleviate the symptoms of depression. 